2. Data collection was over period of time that allowed staff training and familiarisation of the systems as they were being introduced.

3. Our Table 1 showed the results of both EPR systems separately and combined together to allow readers to interpret the data in detail independently. For the Moorfields City Road data, we identified the samples of new patients with a stamp in the medical notes to indicate full documentation of the entry on EPR, based on the discretion of the clinicians. The fact IOP was only recorded in $90 \%$ of the Openeyes entry we speculate was due to it not being a forced choice option.

4. Both electronic and paper data entry in all three sites can be performed by clinicians, trained ophthalmic nurses and technicians. The final entries were all completed by the clinicians. For the paper entry, structured paper new patient proformas were used across three sites.

Following on from our study, one of the centres (Western Eye Hospital) has carried out incremental changes to the EPR documentation in the outpatient glaucoma service. Consent forms are currently scanned and outcome sheets are being made electronic. This programme has now been rolled out to the whole ophthalmology department making it paperless/paperlight.

Whilst we agree electronic records are the "way of the future', the findings of both our study and the findings of Qadir and Kadyan highlight the need for regular and continuous evaluation of the electronic system. This evaluation will ensure safety and reliability in the transition to a 'paperless', or at least a 'paperlight', NHS.

\section{Compliance with ethical standards}

Conflict of interest The authors declare that they have no conflict of interest.

Publisher's note: Springer Nature remains neutral with regard to jurisdictional claims in published maps and institutional affiliations.

\section{References}

1. Qadir M, Kadyan A. Comment on 'How do paper and electronic records compare for completeness? A three centre study'. Eye 2019. https://doi.org/10.1038/s41433-019-0465-4

\title{
Macular toxicity secondary to occupational exposure to gold melting
}

\author{
Hussein Almuhtaseb ${ }^{1} \cdot$ Georgios Tsokolas ${ }^{2} \cdot$ Andrew Lotery $^{3}{ }^{3}$. Assad Jalil ${ }^{4}$
}

Received: 27 April 2019 / Accepted: 2 May 2019 / Published online: 20 May 2019

(c) The Royal College of Ophthalmologists 2019

\section{Case Presentation}

A 60-year-old man was referred to the Vitreoretinal Department of the Manchester Royal Eye Hospital due to

\section{Andrew Lotery}

a.j.lotery@soton.ac.uk

1 Manchester Royal Eye Hospital, Oxford Road, Manchester M13 $9 \mathrm{WL}, \mathrm{UK}$

2 Leicester Royal Infirmary Eye Unit, Leicester Royal Infirmary, Leicester LE1 5WW, UK

3 Southampton Eye Unit, Southampton General Hospital, Tremona Road, Southampton SO16 6YD, UK

4 Manchester Royal Eye Hospital, Oxford Road, M13 9WL Manchester, UK progressive distortion in the left eye. He also complained of increasing difficulty in reading and also of the presence of dark spots in his central vision left worse than the right eye.

The patient's visual symptoms were first attributed to his left cataract but after cataract surgery, he became more aware of a central scotoma.

Of note, the patient mentioned that he worked in the jewelry industry and one of his major tasks was the melting of pure gold. He admitted that occasionally he melted gold without wearing protective goggles, although usually he wore eye protection. He also denied exposure to bright sunlight or looking at the sun for a long time, and he denied any other laser exposure.

On clinical examination, his best corrected visual acuity (BCVA) was 0.7 and 0.9 LogMAR right and left, respectively. Pupillary reflexes were normal and the rest of the 


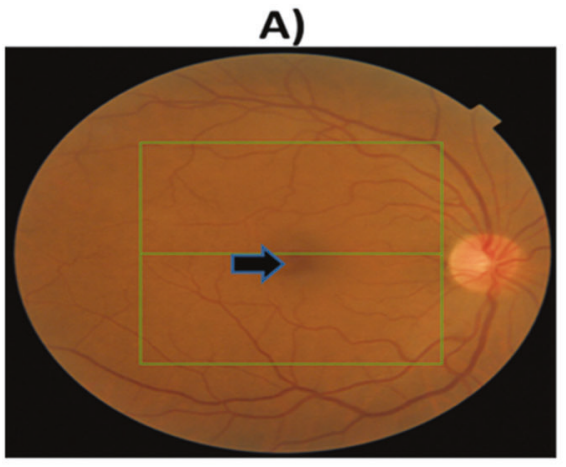

C)

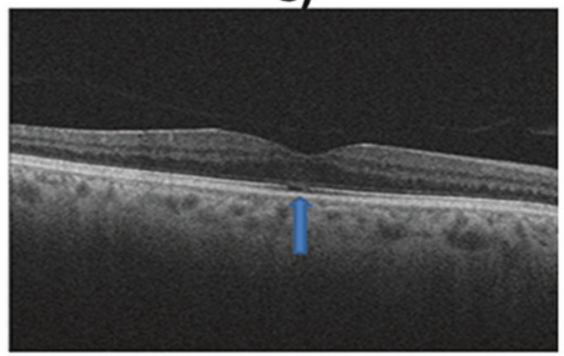

Fig. 1 Top line from left to right: Colour photographs of both fundi. a Right fundus. There are some subtle retinal pigment epithelial (RPE) changes at the right fovea (Black arrow). b Left fundus. The same RPE changes are present but more prominent in the left fovea compared to the right. No other obvious abnormalities are seen in the peripheral retina of either eye. Bottom Line from left to right: Macula OCT

anterior segment examination was normal too. On dilated fundoscopy, the presence of small yellow lesions in both maculae was noted. The findings were more prominent in the left eye compared to the right (Fig. 1a, b). Multimodal imaging including optical coherence tomography (OCT) and fundus autofluorescence (FAF) were obtained with a confocal scanning laser ophthalmoscope (Spectralis HRA-OCT; Spectralis HRA-FAF; Heidelberg Eye Explorer, Version 1.9.17.0, Heidelberg Engineering, Heidelberg, Germany).

A macula OCT demonstrated sub-foveal attenuation, disruption and loss of the ellipsoid layer in both maculae left worse than right (Fig. 1a, b). Furthermore, there were some changes in the inner retinal layers of the left macula as well (Fig. 1c, d). In addition, the left FAF revealed a central area of hypo-autofluorescence which correlated with the central area of disruption and loss of integrity of the subfoveal ellipsoid zone (Fig. 2).

Both optic discs and peripheral retinal did not exhibit any abnormalities.

Based on the history, clinical and multimodal-imaging findings, our hypothesis is macular damage due to occupational exposure during the process of pure gold melting.

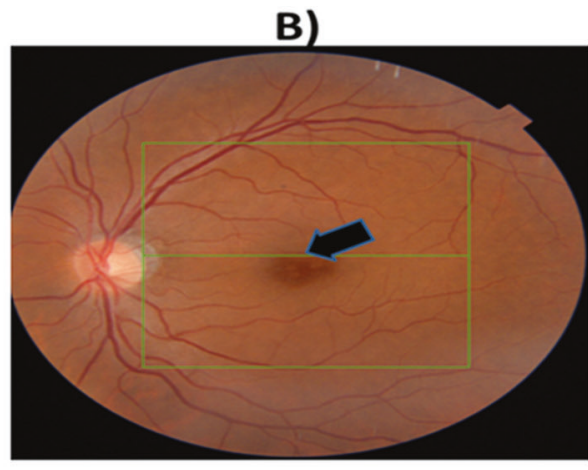

D)

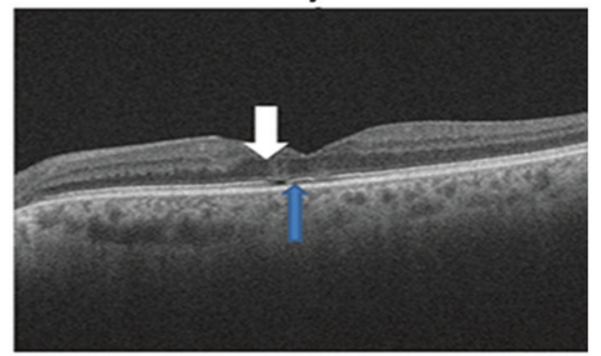

of both eyes. c Right Macula. Note the loss of the ellipsoid as shown by the thin blue arrow d Left Macula. Note the loss and disruption of the ellipsoid zone as shown by the thin blue arrow, which is more prominent in the left macula compared to the right. In addition, the white arrow demonstrates the vertical hyperreflective pillars that traverse the retinal thickness from the ILM to the RPE

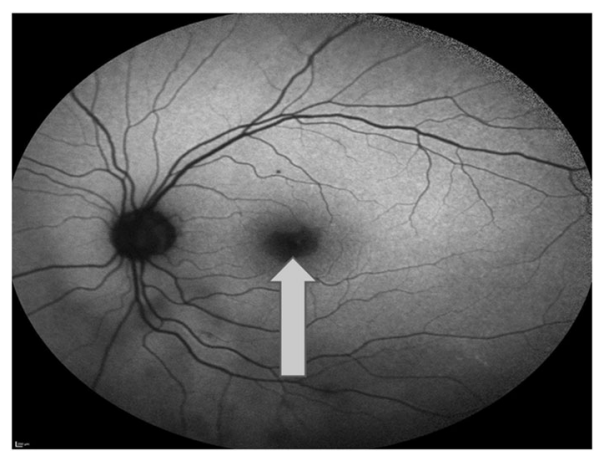

Fig. 2 Fundus autofluorescence of the left eye. The yellow arrow demonstrates a central area of hypo-autofluorescence corresponding to the area of disruption and loss of ellipsoid layer at the level of the left fovea

\section{Discussion}

Light is a form of electromagnetic radiation exhibiting dual wave and particle properties [1]. The wavelengths that can interact with the eye can be divided further in three categories: ultraviolet, visible and infrared. When the light interacts and gets absorbed by a photoreceptor, its particle properties play a pivotal role [1]. 
The electromagnetic spectrum between 400 and $1400 \mathrm{~nm}$ is deemed the retinal hazard region [1]. Three different types of retinal damage can occur due to light toxicity: photothermal, photochemical and photomechanical [1]. We postulate that our patient developed a photochemical injury due to exposure to the melting process of gold.

When trying to melt pure gold in powder form, it has been found that powdered gold is more sensitive and reflective in the infrared region from 1060 to $1090 \mathrm{~nm}$ [2]. A specific laser system called selective laser melting (SLM, MTT Technologies Group SLM 100) has been designed to produce infrared wavelength in the aforementioned region [2]. The wavelengths emitted by the SLM machine fall within the retinal hazard region and thus, they can potentially be harmful to the human retina.

We postulate that these infrared waves might have reflected onto the surface of the powdered melting gold, and hit the patient's macula while the patient was gazing directly without protective goggles. As the patient had repeatedly done so, there may have been a cumulative aggregation of photochemical retinal toxicity that eventually led to the manifestation of the retinal pigment epithelial (RPE) changes and the OCT and FAF findings described above. These are very typical findings of photochemical injury [1]. They provide a logical explanation for the patient's central visual disturbances and distortion mainly in his left eye.

Another possible type of damage that might have contributed was photothermal injury due to increase in the temperature of the retina cells, which subsequently resulted in the denaturation of retinal proteins, and loss of their tertiary structure with simultaneous liquefaction of the photoreceptor cell membrane [1]. Indeed, during gold melting, the increase in thermal energy produced might have penetrated the eye and reached the macular area while the patient was working and gazing directly without protective goggles.

Absorption of photothermal energy is thought to occur by one of three pigments: melanin located primarily in the melanosomes of the retinal pigment epithelium (RPE) and melanocytes of the choroid, xanthophyll located primarily in Muller cells and neurosensory retina, and haemoglobin in the blood vessels of the neurosensory retina and choroid. Melanin, the most effective absorber, is located primarily in the RPE. Therefore, an eye with an abundance of melanosomes, as in a heavily pigmented fundus (as is the case of our patient who is dark skinned), will more readily absorb photothermal energy. Following the application of laser to the retina and RPE, histological evidence of thermal damage is seen initially at the level of both the RPE and photoreceptors [3]. Therefore, both photochemical and photothermal damage from gold melting may have contributed to the ocular damage seen in this patient.

\section{Conclusion}

To the best of our knowledge, this is the first reported case of macular light toxicity secondary to the process of gold melting, and highlights the importance of wearing protective goggles when undertaking this process.

Ophthalmic exposure to radiation during gold melting should be considered an occupational hazard. The jewelry industry should mandate adequate safety precautions in terms of protective goggles, which block at least the infrared region from 1060 to $1090 \mathrm{~nm}$. Otherwise further irreversible visual loss may occur in other workers.

\section{Compliance with ethical standards}

Conflict of interest The authors declare that they have no conflict of interest.

Publisher's note: Springer Nature remains neutral with regard to jurisdictional claims in published maps and institutional affiliations.

\section{References}

1. Youssef PN, Sheibani N, Albert DM. Retinal light toxicity. Eye (Lond). 2011;25:1-14.

2. Khan M, Phill Dickens P. Selective laser melting (SLM) of pure gold for manufacturing dental crowns. Rapid Prototyp J. 2014;20:471-9.

3. Green WR, Robertson DM. Pathologic findings of photic retinopathy in the human eye. Am J Ophthalmol. 1991;112:520-7. 